\title{
Overexpression of Astrocyte Elevated Gene-1 (AEG-1) in Cervical Cancer and its Correlation with Angiogenesis
}

\author{
Jian-Qin Yu, Qing Zhou, Hua Zhu, Fei-Yun Zheng, Zhi-Wen Chen*
}

\begin{abstract}
Objectives: To explore the expression of astrocyte elevated gene-1 (AEG-1) in cervical cancer and analyze its correlation with microvascular density (MVD), nuclear factor kappaB (NF-kB p65) and vascular endothelial growth factor (VEGF). Materials and Methods: Immunohistochemical MaxVision method was adopted to detect the expression level of AEG-1, NF-kB p65 and VEGF in 45 samples of invading cervical cancer and 12 samples of cervicitis from The First Affiliated Hospital of Wenzhou Medical University. Tumor microvascular endothelial marker CD34 combined with Weidner was used to determine the MVD in cervical cancer tissue. The positive expression and staining conditions of AEG-1, NF-kB p65 and VEGF in cervical cancer tissues were observed under a light microscope. Correlations between expression of AEG-1 protein and those of NF-Kb p65 and VEGF, as well as MVD, were analyzed using Pearson correlation. Results: The expression levels of AEG-1 were $0.186 \pm 0.043$ in cervical cancer and $0.051 \pm 0.002$ in chronic cervicitis $(p<0.01)$. Moreover, expression of AEG-1 was related to vascular invasion and lymphatic metastasis of cervical cancer $(p<0.01)$, but not with age of the patients, differentiation degree, tumour size, pathological type and parametrial infiltration $(p>0.05)$. Pearson correlation analysis showed that the expression of AEG-1 was linked with NF-kB p65 ( $r=0.501, p=0.000)$, VEGF $(\mathrm{r}=\mathbf{0 . 7 1 8}, p=0.000)$ as well as MVD in cervical cancer tissue $(\mathrm{r}=\mathbf{0 . 8 1 5}, p=\mathbf{0 . 0 0 0})$. Conclusions: AEG-1 is highly expressed in cervical cancer and promotes angiogenesis, which might be related to the fact that AEG-1 activating the signal pathway of NF-kB could up-regulate the level of VEGF expression.
\end{abstract}

Keywords: Cervical cancer - astrocyte elevated gene-1 - angiogenesis - nuelear factor kappaB - microvascular density

Asian Pac J Cancer Prev, 16 (6), 2277-2281

\section{Introduction}

Cervical cancer is one of the most common malignant tumors in female and has a tendency to young people in recent years, many of which have bad biological behaviors at an early stage such as early invasion and metastasis, causing unfavorable prognosis (Ferlay et al., 2008; Miglierini et al., 2014). Study after study have shown the microvascular proliferation plays an important role in promoting invasion and metastasis of cervical cancer and related to poor prognosis of cervical cancer (Biedka et al., 2012). Adtrocyte elevated gene-1 (AEG-1) is a kind of gene with high expression in primary human fetal astrocytes (PHFA) induced by human immunodeficiency virus (HIV) and tumor necrosis factor $\alpha(\mathrm{TNF} \alpha)$, becoming a hot issue in the field of cancer research presently.

With the research progress of molecular biology, molecular targeted therapy has become the forth method for treating malignant tumors after the application of surgery, chemotherapy and radiotherapy. A large number of studies have shown that AEG-1 has the feature of oncogene and is closely associated with the occurrence and development of tumors, such as nasopharyngeal carcinoma, esophageal cancer, breast cancer, lung cancer, liver cancer and renal cell carcinoma (Chen et al., 2010; Yoo et al., 2011; Gu et al., 2012; Claudio et al., 2012; Liu et al., 2014).

AEG-1 can activate the signal pathway of NF-kB. The activated NF-kB, as regulatory factor in the upstream, can un-regulate the expression of the multiple targeted genes in the downstream, thus producing a variety of functional protein of regulating biological functions of tumor cells such as cell metabolism, survival, apoptosis and angiogenesis (Endad et al., 2009). Therefore, study on angiogenesis-associated regulatory factors and their mechanism have great significance for clinical diagnosis, treatment and prognosis of cervical cancer. This study was intended to use tumor microvascular endothelial marker CD34 and immunohistochemical MaxVision method to detect the expressions of AEG-1, NF-kB p65 and VEGF in cervical cancer tissues and analyze the correlation between AEG-1 and angiogenesis of cervical cancer. 


\section{Materials and Methods}

\section{Samples}

This study was approved by Ethics Committee of The First Affiliated Hospital of Wenzhou Medical University. A total of 45 samples with cervical cancer were collected from patients with invasional cervical cancer in The First Affiliated Hospital of Wenzhou Medical University from Juny 2011 to Jan. 2014, who were aged from 26 to 61 years old with the median age of 45 years. There were 20 cases with lymph node metastasis, 15 cases with vascular invasion, 9 with parametrial infiltration, 12 with the diameter of tumor $\geq 4 \mathrm{~cm}, 17$ with low differentiation, 16 with middle differentiation and 12 with high differentiation and 7 with squamous-cell carcimoa and 38 cases with adenocarcimoa. All patients were not performed with radiotherapy, chemotherapy and other special therapies preoperatively. In addition, 12 samples with chronic cervicitis were selected as for comparison. All patients agreed to participate in this study and signed the informed consent form.

\section{Reagents}

Condensed rabbit anti-AEG-1 polyclonal antibody, NF-Kb p65 (B7172) rabbit polyclonal antibody and VEGF rabbit polyclonal antibody were bought from Beijing bo orson biological technology co., LTD, ANBO Company and Wuhan Boster Biological Technology, Ltd, respectively. Rat anti-CD34 monoclonal antibody (MAB0034) and MaxVision second antibody kit were purchased from Fuzhou Maixin Biotechnology Development Co., Ltd.

\section{Immunohistochemistry}

Immunohistochemical MaxVision method was used to detect the expressions of AEG-1, NF-kB p65 and VEGF proteins. The 3 5- $\mu$ m paraffin-embedded tissue sections were conducted with dewaxing, hydration and thermal remediation of antigen in citric acid. After treated by $3 \% \mathrm{H}$ of $\mathrm{H} 2 \mathrm{O} 2$ and washed by PBS, every section was added with $50 \mathrm{uL}$ of primary antibody working solution, incubated at room temperature for $3 \mathrm{~h}$, washed by PBS again for three times, and then added with $50 \mathrm{uL}$ of MaxVision second antibody working solution for incubation for $30 \mathrm{~min}$. Sections was developed by DAB after rinsing, redyed by hematoxylin and sealed by neutral balsam. PBS was regarded as negative control. Positive staining of AEG1, NF-kB p65 and VEGF in the cytoplasm presented claybank. Five high power fields of the strongest positive expression in gross target volume was photographed with digital camera and put into Image-Pro 6.0 in order to mark off accurate positive area and measure the mean density (MD). Each picture was measured 3 times. The mean of 3 integrated optical density values was considered as the expression intensity of protein in this field, and the mean of MD in the five visual fields in the same section was as the average expression intensity of protein in single sample tissue in the section.

\section{Determination of microvascular density}

Weidner method was used to determine the microvascular density (MVD) in cervical cancer tissue and the method was as described below: CD34 presenting claybank particles were labeled as the standard of vascular endothelium. The highest area of MVD distribution in the section was selected under light microscope $(\times 100)$. The positive microvessel quantity (MVQ) stained by CD34 in five different visual fields was counted under light microscope $(\times 400)$ to calculate the average as the value of MVD. Positive single vascular endothelial cell or vascular endothelial cell cluster which separated from adjacent capillaries distinctly was deemed as the independent microvessel, including branch vessels of unconnected structure. Vessels with the diameter $\geq 8$ red blood cell, vessels with thick smooth muscle and vessels with sclerotic or necrotic area were not included in counter range.

\section{Statistical data analysis}

SPSS 15.0 software package was used for data analysis. The measurement data of the experiment was expressed as " $x \pm s$ " and the comparison of indexes between cervical cancer and chronic cervicitis was analyzed using $t$ test. The correlation between AEG-1 expression and VEGF, NF-kB p65 and MVD was analyzed using Pearson Correlation. A value of $p<0.05$ was considered to be statistical significant.

\section{Results}

The expression of AEG-1 in cervical cancer and chronic cervicitis

AEG-1 protein was highly expressed in cervical cancer. The expression quantity of AEG- 1 was $(0.186 \pm 0.043)$ in cervical cancer and $(0.051 \pm 0.002)$ in chronic cervicitis and there were statistical significance between them $(p<0.01)$. AEG-1, NF-kB p65 and VEGF were expressed positively in cervical cancer tissue, the cytoplasm presented claybank particles and cell nucleus developed blue by hematoxylin, as shown in Figure 1, 2 and 3.

The relationship between AEG-1 expression and clinicopathological features

The expression of AEG-1 was related to vascular invasion and lymphatic metastasis of cervical cancer $(p<0.01)$, but not associated with age of the patients, differentiated degree, size, pathological type and

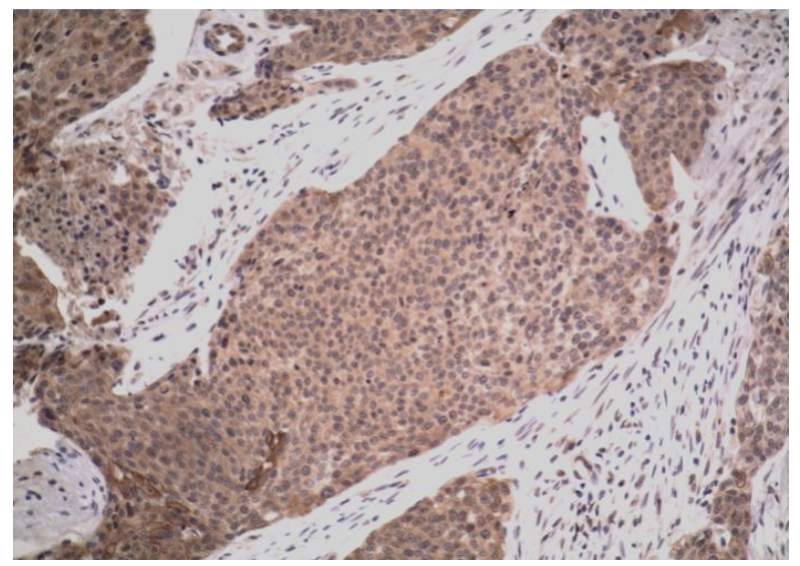

Figure 1.AEG-1 were Expressed Positively in Cervical Cancer Tissue $(\times 400)$ 


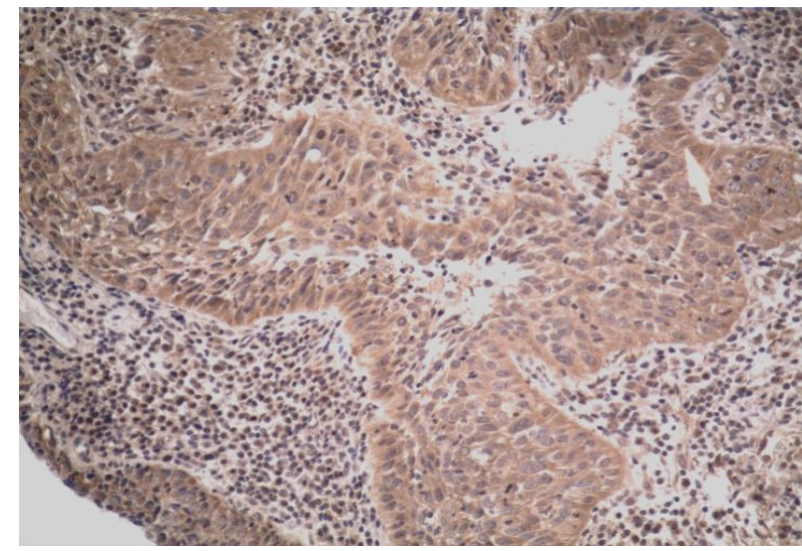

Figure 2. NF-Kb p65 were Expressed Positively in Cervical Cancer Tissue $(\times 400)$

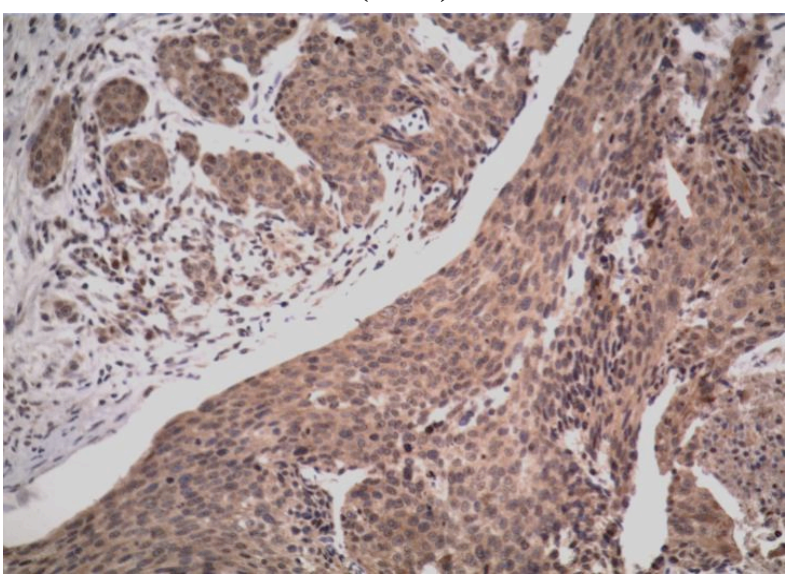

Figure 3. VEGF were Expressed Positively in Cervical Cancer Tissue $(\times 400)$

Table 1. The Relationship between AEG-1 Expression and Clinicopathological Features $(\bar{x} \pm s)$

\begin{tabular}{lrcc}
\hline Items & $\mathrm{n}$ & AEG-1 & $p$ value \\
\hline Age (years) & & & \\
$\quad<45$ & 14 & $0.198 \pm 0.035$ & $p>0.05$ \\
$\quad \geq 45$ & 31 & $0.185 \pm 0.031$ & \\
Differentiated degree & & & \\
$\quad$ High+middle differentiation & 28 & $0.173 \pm 0.048$ & $p>0.05$ \\
$\quad$ Low differentiation & 17 & $0.189 \pm 0.034$ & \\
Diameter of tumor & & & \\
$\quad<4$ cm & 33 & $0.168 \pm 0.042$ & $p>0.05$ \\
$\quad \geq 4$ cm & 12 & $0.174 \pm 0.037$ & \\
Pathological type & & & \\
$\quad$ Adenocarcinoma & 38 & $0.186 \pm 0.023$ & $p>0.05$ \\
$\quad$ Squamous carcinoma & 7 & $0.181 \pm 0.019$ & \\
Vascular invasion & & & \\
$\quad$ Yes & 15 & $0.197 \pm 0.041$ & $p<0.01$ \\
$\quad$ No & 30 & $0.161 \pm 0.034$ & \\
Parametrial infiltration & & & \\
$\quad$ Negative & 36 & $0.169 \pm 0.045$ & $p>0.05$ \\
$\quad$ Positive & 9 & $0.199 \pm 0.047$ & \\
Lymphatic metastasis & & & \\
$\quad$ Yes & 20 & $0.197 \pm 0.041$ & $p<0.01$ \\
\hline
\end{tabular}

parametrial infiltration $(p>0.05)$.

Correlation between AEG-1 and the expressions of VEGF, $N F-k B$ p65 as well as MVD in cervical cancer tissue

Pearson Correlation analysis showed that the expression of AEG-1 in cervical cancer was in positive

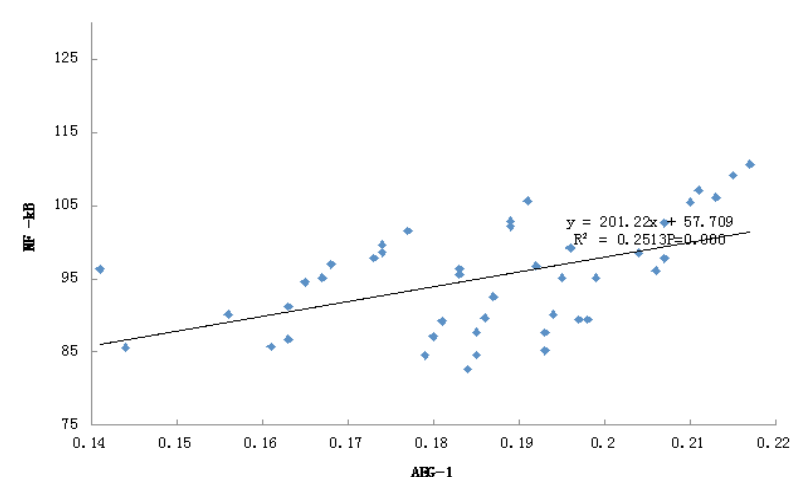

Figure 4. Correlation between the Expression of AEG-1 and NF-Kb p65

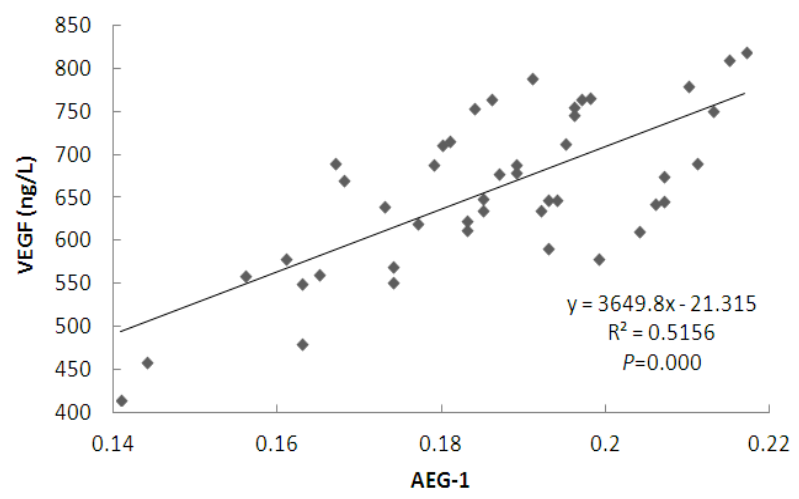

Figure 5. Correlation between the Expression of AEG-1 and the Expression of VEGF

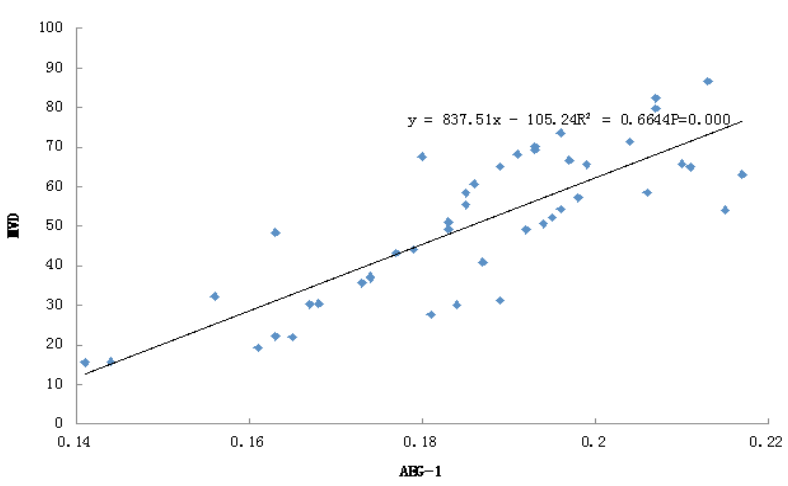

Figure 6. Correlation between the Expression of AEG-1 and the Expression of MVD in Cervical Cancer Tissue

correlation with NF-kB p65 ( $\mathrm{r}=0.501, p=0.000)$, VEGF $(\mathrm{r}=0.718, p=0.000)$ as well as MVD in cervical cancer tissue $(\mathrm{r}=0.815, p=0.000)$. (Figure 4, 5 and 6).

\section{Discussion}

Cervical cancer is the third most common malignant tumor and the fourth leading cause of cancer-related death among women worldwide (Miglierini et al., 2014; Salomon-Perzynska et al., 2014). Its pathophysiological mechanism has been the focus of scholars' attention (Parkin et al., 2001; Mori et al., 2001). Tumor angiogenesis is closely associated with the development, metastasis and prognosis of solid tumor, which has been generally recognized (Fidler et al., 1994; Liu, et al., 2012). Tumor tissue obtains rich nutrients from new vessels and flows out through them, so they have invasion ability and metastasis potential. In addition, multiple vascular growth factors 
secreted from tumor cell and macrophage beside tumor tissues play an important role in angiogenesis of tumors. Therefore, study on those vascular growth factors is of great significance for revealing the law of the occurrence and development of the tumors. Presently, most researches hold that angiogenesis is not only the initial period of cervical cancer but also an important process of malignant change transformed from normal epithelial.

AEG-1 is a new oncogene discovered in recent years, which can unregulate the expressions of HIF1- $\alpha$ and MMP1 in tumor tissues, thus promoting tumor angiogenesis. AEG-1 is a targeted molecule in the downstream of signal transduction pathway of Ras/P12K/Akt/c-Myc which involves in multiple links of the occurrence and development of normal cells through regulating multiple signaling pathways such as PI3K/Akt, NF-kB, Wnt/ $\beta$ catenin and MAPK as well as mediates the autophagy activity and chemotherapy resistance (Emdad et al., 2009; Yoo et al., 2011). Hence, AEG-1, as an oncogene expressed in a variety of malignant tumors, provides a new idea for molecular targeted drugs in the treatment of malignant tumors in clinc. Emdad et al's study proved that AEG-1 could increase tumor MVD, and its overexpression could enhance the expressions of angiogenin-1 (Ang-1) and matrix metalloproteinase-2 (MMP-2) and promote angiogenesis of tumor tissues.

$\mathrm{NF}-\mathrm{kB}$ was a kind of protein factor found in samples of B lymphocyte and plasma cell nucleus by Sen in 1986. The over-expressed AEG-1 can activate the signal pathway of $\mathrm{NF}-\mathrm{kB}$ and the activated NF-kB, as the regulatory factor in the upstream, can lead to the up-regulation of multiple targeted genes in the downstream. As one member of transcription factors of NF-kB, heterodimer of p65 participates in the transcriptional regulation of a variety of cellular biological functions such as cell metabolism, survival, apoptosis and angiogenesis of tumor (Li, et al., 2011). When AEG-1 acts on p65, activated IKK in the cytoplasm has kappaB inhibit the phosphorylation of IkB, consequently weakening the inhibitory effect of $\mathrm{IkB}$ on p65 in the cytoplasm and increasing retroposition of NF$\mathrm{kB}$ p65. In the cell nucleus, AEG-1 was the bridge binding p65 with CBP and further regulates the transcriptional level of the target gene for NF-kB (Sarkar et al., 2008). At present, researches on explore the correlation between AEG-1 and invasion ability of cervical cancer have been seldom reported from the perspective of AEG-1 regulating the angiogenesis of cervical cancer through the signal pathway of NF-kB.

In this study, vascular endothelial marker CD34 was used for detecting MVD in tumor tissues and immunohistochemistry for detecting the expression levels of AEG-1, NF-kB p65 and VEGF in cervical tissues in order to analyze the correlation between AEG-1 and MVD, NF-kB p65 and VEGF expression and the relationship between AEG-1 expression and clinicopathological features of cervical cancer. The results showed that AEG-1 was highly expressed in cervical cancer tissue $(0.186 \pm 0.043)$ when compared with chronic cervicitis $(0.051 \pm 0.002)$, and there was statistical significance $(p<0.01)$, showing that AEG-1 might participate in the occurrence and development of cervical cancer. Pearson
Correlation analysis showed that the expression of AEG-1 in cervical cancer was in positive correlation with NF-kB p65 ( $\mathrm{r}=0.501, p=0.000)$, VEGF $(\mathrm{r}=0.718$, $p=0.000)$ as well as MVD in cervical cancer tissue $(\mathrm{r}=0.815, p=0.000)$, which was consistent with the partial results of Liu et al's study (Liu et al., 2012). However, Liu didn't analyze the correlation between AEG-1 and NF-kB p65, VEGF. Additionally, AEG-1 expression was related to vascular invasion and lymphatic metastasis of cervical cancer $(p<0.01)$, but not associated with age of the patients, differentiated degree, size, pathological type and parametrial infiltration $(p>0.05)$.

In conclusion, AEG-1 is highly expressed in cervical cancer. AEG-1 improves the invasion and metastasis ability through enhancing the ability of angiogenesis of cervical cancer, which might be related to the fact that AEG-1 activating the signal pathway of NF-kB upregulates the level of VEGF expression.

\section{References}

Biedka M, Makarewicz R, Maeszalek A, et al (2012). Labeling of microvessed density, lymphatic vessel density and potential role of proangiogenic and lymphatic factors as a predictive/ prognostic factors after radiotherapy in patients with cervical cancer. Eur J Gynaecol Oncol, 33, 399-405.

Chen W, Ke Z, Shi H, et al (2010). Overexpression of AEG-1 in renal cell carcinoma and its correlation with tumor nuclear grade and progression. Neoplasma, 57, 522-9.

Claudio Luparello, Alessandra Longo, Marco Vetrano (2012). Exposure to cadmium chloride influences astrocyte-elevated gene-1 (AEG-1) expression in MDA-MB231 human breast cancer cells. Biochimie, 94, 207-13.

Emdada L, Lee SG, Su ZZ, et al (2009). Astrocyte elevated gene-1 (AEG-1) functions as an oncogene and regulates angiogenesis. Proc Natl Acad Sci USA, 106, 21300-5.

Fidler IJ, Ellis LM, (1994). The implications of angiogenesis for the biology and therapy of cnacer metastasis. Cell, 79, 185-8.

Ferlay J, Shin HR, Bray F, et al (2010). Estimates of worldwide burden of cancer in 2008: GLOBOCAN 2008. Int J Cancer, 127, 2893-917.

Gu Wenwen, Cao Yongbing, Jiang Yuanying, et al (2012). Astrocyte elevated gene 1 as a multifunctional regulator in cancer progression. Acad J Sec Mil Med Univ, 33, 668-72.

Li C, Li R, Song H, et al (2011). Significance of AEG-1 expression in correlation with VEGF, microvessel density and clinicopathological characteristics in triple-negative breast cancer. Surg Oncol, 103, 184-92.

Liu L, Liao H (2014). The research progress of AEG-1 in head and neck cancer. Med Recapitulate, 20, 813-6.

Liu X, Wu C, Xu H et al (2012). Expression of AEG-1and the CD105 marked microvessel density in esoghageal squamous cell carcinoma and their correlation with metastasis and prognosis. J Fujian Med Univ, 46, 24-7.

Long M, Dong K, Gao P, et al (2013). Overexpression of astrocyte-elevated gene- 1 is associated with cervical carcinoma progression and angiogenesis. Oncol Rep, 30, 1414-22.

Mori M, Sagae S (2011). Resent progress in epidemiologic research of uterine cancer. Gan To Dagako Ryoho, 28, 174-8.

Miglierini P, Malhaire JP, Goasduff G, et al (2014). Cervix cancer brachytherapy: high dose rate. Cancer Radiother, 18, 452-7.

Parkin DM, Bray F, Ferlay J, et al (2001). Estimating the world cancer burder: Globocan 2002. Int J Cancer, 94, 153-6.

Su ZZ, Kang DC, Chen Y, et al (2002). Identification and cloning 

of human astrocyte genes displaying elevated expression after infection with HIV-1 or exposure to HIV-1 envelope glycoprotein by rapid subtraction hybridization, $\mathrm{RaSH}$. Oncogene, 21, 3592-602.

Su ZZ, Chen Y, Kang D, et al (2003). Customized rapid subtraction hybridization (RaSH) gene microarrays identify overlapping expression changes in human fetal astrocytes resulting from human immunodeficiency virus-1 infection or tumor necrosis factor-alpha treatment. Gene, 306, 67-78.

Sarkar S, Park ED, Emdad L, et al (2008). Molecular basis of nuclear factor-kappaB activation by astrocyte elevated gene1. Cancer Res, $\mathbf{6 8}, 1478-84$.

Salomon-Perzynska M, Perzynski A, Rembielak-Stawecka B, et al (2014). VEGF--targeted therapy for the treatment of cervical cancer-literature review. Ginekol Pol, 85, 461-5.

Yoo BK, Emdad L, Lee SG, et al (2011). Astrocyte elevated gene-1 (AEG-1): A multifunctional regulator of normal and abnormal physiology. Pharmacol Ther, 130, 1-8. 